\title{
EL USO DE UNA HERRAMIENTA RIA EN APOYO A LA TOMA DE DECISIONES IMPLEMENTADA EN UN PROYECTO DE IMPACTO SOCIAL: CASO TABASCO
}

\author{
THE USE OF AN RIA TOOL IN SUPPORT OF DECISION-MAKING IMPLEMENTED \\ IN A SOCIAL IMPACT PROJECT: TABASCO CASE
}

\begin{abstract}
Gilberto Murillo-González ${ }^{1}$; Jorge Omar Vázquez-Romero ²; Francisco Javier Jiménez-Tecillo ${ }^{3}$.
1. Universidad Juárez Autónoma de Tabasco, División Académica de Informática y Sistemas, Tabasco, México. gilberto.murillo@ujat.mx; gmurillo76@gmail.com.

2. Universidad Juárez Autónoma de Tabasco, División Académica de Ciencias Básicas, Tabasco, México. omar.vazquez@ujat.mx.

3. Universidad Juárez Autónoma de Tabasco, División Académica de Ciencias Económico Administrativas, Tabasco, México.tecillo3302@gmail.com.

*Correspondencia del Autor: Gilberto Murillo-González, correo electrónico: gilberto.murillo@ujat.mx.

\section{RESUMEN}

La presente investigación, tiene como finalidad establecer el uso de un sistema de información para la Instancia Operadora Estatal (IOE) del Proyecto México Conectado (PMC) en el Estado de Tabasco, con el objetivo de mejorar el registro, control y acceso a la información de los sitios y espacios públicos en relación a las diversas actividades que se realizan de forma manual para su identificación, así como el almacenamiento de la información de las estructuras variadas, lo que genera inconsistencias, pérdida de tiempo y falta de organización para realizar la toma de decisiones adecuadas. Para el diseño y desarrollo, se optó en realizar una aplicación RIA y utilizar una metodología de desarrollo SCRUM, haciendo uso de nuevas técnicas en el ambiente. web. Como resultado se obtuvo mejoras sustanciales para gestionar la información de los 6,763 sitios propuestos para ser beneficiados con internet de alta velocidad y una base de datos unificada y homogénea.
\end{abstract}

Palabras clave: Sistema de información; RIA; metodología ágil; México conectado; desarrollo móvil.

Cómo citar:

Murillo-González, Gilberto; Vázquez-Romero, Jorge Omar; Jiménez-Tecillo, Francisco Javier. (2021). EL USO DE UNA HERRAMIENTA RIA EN APOYO A LA TOMA DE DECISIONES IMPLEMENTADA EN UN PROYECTO DE IMPACTO SOCIAL: CASO TABASCO. Revista de Investigaciones Universidad del Quindio, 33(1), 140-153. https://doi.org/10.33975/riuq.vol33n1.517 


\begin{abstract}
The purpose of this research is to establish the importance of using an information system for the State Operator Instance (IOE) of the Mexico Connected Project (PMC) in the State of Tabasco, with the aim of improving the registration, control and access to the information of the sites and public spaces in relation to the various activities that are carried out manually for identification, as well as the storage of the information is kept in various archives, which contain varied structures, which generates waste of time and lack of organization at the time of making appropriate decisions for the project. For the development of the solution proposal, it was decided to make an RIA application and use the SCRUM development methodology, using new programming techniques for web environments. As a result, substantial improvements were obtained from the system that allows managing the information of the 6,763 sites proposed to be benefited with high-speed internet in the State, complying with each of the requirements associated with it; Scalable and easy to use for the user. In addition to a unified and homogeneous database.
\end{abstract}

Keywords: Information system; RIA; agile methodology; connected Mexico; mobile development.

\section{INTRODUCCIÓN}

La Web en la que actualmente interactuamos es un universo que lo integran diversos sitios, aplicaciones y páginas interconectadas en todas partes del mundo. En este inmenso número de herramientas se contemplan una variedad de contenidos interactivos, conformado en su gran mayoría por datos, fotos, audios y videos. Alo largo del tiempo, las tecnologías web han evolucionado hasta permitir que los desarrolladores puedan crear nuevas e increíbles experiencias en la web. Con el crecimiento acelerado que la web ha tenido desde su creación en 1989, los esfuerzos continuos de una comunidad abierta a la transformación de los paradigmas iniciales se reflejan en la creación de nuevas tecnologías, tales como: HTML5, CSS3, WebGL, Ajax, RIA y entre otras, las cuales permiten la construcción de nuevos entornos altamente dinámicos para los usuarios. (Histinf.blogs.upv.es, 2016).

Adobe Systems (2008), explica que las Rich Internet Applications (RIA) es el término con el que define a las aplicaciones web que se comportan del mismo modo que las aplicaciones tradicionales de escritorio, pero con la capacidad de ser ejecutadas en cualquier navegador web. A diferencia de las páginas web clásicas, estas aplicaciones están orientadas a realizar tareas complejas que tradicionalmente habían sido exclusivas de los programas de escritorio, aportando una gran flexibilidad y potencia al usuario final.

El uso de herramientas tecnológicas, como las aplicaciones RIA, base de datos y ambientes web, permiten contribuir a la evolución de la administración de proyectos, desarrollando canales electrónicos de comunicación entre la información que se procesa al instante, hasta la información estandarizada de carácter histórico, en bancos único de datos para su seguimiento y control. (Histinf.blogs.upv.es, 2016).

En la actualidad las instituciones gubernamentales se enfrentan al reto de almacenar y administrar toda la información que generan. Los esfuerzos por utilizar bancos de datos únicos de almacenamientos y el uso de herramientas de Tecnologías de la Información (TI) para simplificar proceso y actividades son necesarias para la consolidación de proyectos de carácter nacional; dentro de este escenario y durante el desarrollo del Proyecto México Conectado (PMC), iniciativa del gobierno federal para brindar internet de alta velocidad a sitios $y$ 
espacios públicos en todo el país, se contempla la creación de grandes volúmenes de información, producto del cumplimiento de sus cuatro fases planeación, licitación, implementación y operación. Estas fases necesitan información veraz para la identificación del universo final de sitios propuestos por cada entidad. En el caso particular de acuerdo con la (ICN, 2014) para el Estado de Tabasco, el número de sitios propuestos son 7,272 sitios distribuidos en toda la entidad.

El propósito que se persigue en la presente investigación es la identificación de las diversas estrategias para el desarrollo de una aplicación, basada en tecnología RIA, para la Instancia Operadora Estatal de Tabasco (IOE), la cual sirva como una herramienta para el control y almacenamiento de los registros, inventarios, estadísticas y acceso a la información producida durante el desarrollo de las diversas fases del Proyecto México Conectado (PMC) en el estado de Tabasco, las cuales permitan coadyuvar en la integración de los expedientes electrónico de cada sitio propuesto al momento de su revisión y aplicación de los criterios de elegibilidad de acuerdo a lo establecido por la CSIC, en los Lineamientos del Proyecto México Conectado, (2013a), para el Estado de Tabasco en sus diversos municipios.

El presente artículo se divide en 4 secciones. En la primera se establecen las características generales del proyecto y su interacción en el Estado de Tabasco, en conjunto con los tres órdenes de gobierno, la Universidad de Guadalajara y la Universidad Juárez Autónoma de Tabasco. La segunda sección describe la metodología empleada en la selección e identificación de los sitios y espacios públicos propuestos a recibir conectividad en el Estado de Tabasco. En la Tercera sección se muestran los resultados obtenidos y su integración en la aplicación RIA, para el control, resguardo y seguimiento de la información procesada en las diversas fases del proyecto en apoyo a la toma de decisión y por último, la cuarta sección presenta las conclusiones.

\section{CARACTERÍSTICAS GENERALES DEL PROYECTO MÉXICO CONECTADO Y SU INTEGRACIÓN EN EL ESTADO DE TABASCO.}

En la Universidad Juárez Autónoma de Tabasco (UJAT), se desarrollan diversos proyectos con fines y propósitos distintos, enfocados al servicio de la sociedad. Uno de estos proyectos tiene la connotación de la aplicación de políticas publicas, bajo la iniciativa que contribuye a garantizar el derecho constitucional de acceso al servicio de internet de banda ancha, de acuerdo con lo establecido en el artículo 6to de la constitución. Iniciativa que se encuentra insertada dentro del marco institucional del Proyecto México Conectado, coordinado por la Secretaría de Comunicaciones y Transporte (SCT), bajo la dirección de la Coordinación de la Sociedad de la Información y el Conocimiento (CSIC). (CDM, 2014). Piña Gutierrez, (2018), establece en su informe de actividades, derivado de las actividades realizadas por la UJAT, uno de los tantos logros alcanzados en el periodo 2014 -2015 por esta casa de estudio en los ámbitos del fortalecimiento y reducción de la brecha digital en la entidad, fue la participación activa en la implementación de políticas publicas vinculando las capacidades físicas, intelectuales y de formación de la comunidad universitaria en proyectos de alto impacto social en materia de las telecomunicaciones y cambio cultural tecnológico fortalecidos por la participación de los tres ordenes de gobierno.

De acuerdo con la estructura y normas establecidas en el proyecto (ICN 2014; CDM 2014), la UJAT participa dentro del Proyecto México Conectado, como la Instancia Operadora Estatal (IOE), la cual tiene la responsabilidad de procesar y analizar la información proveniente de los Comité Técnico de Conectividad (CTC) y del Comité del Uso y Aprovechamiento de la Conectividad Social (CUACS), a través de los procedimientos establecidos por la Secretaría de Comunicaciones y Transporte (SCT), por conducto de la Coordinación de la Sociedad 
de la Información y el Conocimiento (CSIC). (CDM, 2014). Esta responsabilidad estable tener acceso a información de cada dependencia, organismos, institución y responsables de sitios, inmuebles e infraestructura propuestos para la implementación del proyecto en la entidad, para lo cual es necesario establecer mecanismos de automatización y control que permita analizar, revisar, validar, procesar y homogeneizar la información, para poder ser autorizada por parte de los órganos de control del PMC. (CSICa, 2018).

Asimismo dentro de algunas funciones convergentes por parte de la IOE, se encuentra la generación de diversos levantamiento de información especifica del tipo de infraestructura de telecomunicación existente en la entidad, elaborar diversos inventario del tipo de inmuebles, sitios y activos públicos disponibles en los tres ordenes de gobierno, así como el estado que guarda, para la compilación de las base de datos central, que contendrá el numero de sitios a conectar en la entidad, identificando entre algunas acciones, las aplicaciones que estos utilizan, de acuerdo a los formatos establecidos por la Coordinación de la Sociedad de la Información y el Conocimiento (CSIC) o de la Instancia Coordinadora Nacional (ICN). (CDM, 2014).

\section{METODOLOGÍA}

La presente investigación, tuvo como propósito determinar la aplicación de herramientas cualitativas y cuantitativas de gestión administrativa, para la construcción de estrategias en la implementación de un sistema de información tipo RIA para agilizar la toma de decisión del Proyecto México Conectado en el Estado de Tabasco. Además, se realizó bajo un diseño exploratorio descriptivo, apoyado en la investigación de campo y documental, la integración de la información referente a la población de estudio, la cual fue integrada por toda la información proporcionada por las dependencias participantes, concentrada en la definición e identificación de los sitios y espacios públicos adscritos a los sectores de educación, salud y gobierno. A partir de ello, se obtuvo el universo de 6,763 inmuebles propuestos a recibir internet gratuito.

\subsection{Proceso de recopilación de la información de los sitios propuesto a recibir conectividad por parte del Proyecto México Conectado en el Estado de Tabasco}

La información del Proyecto México Conectado, tiene su origen en la definición y planteamiento del universo propuesto de sitios y espacios públicos a conectar en el Estado de Tabasco, así como los activos disponibles en materia de telecomunicaciones y sus necesidades específicas, mediante la dirección de la Mesa de Coordinación (MC), la cual establece los criterios de aprobación y selección del ancho de banda requerido, la geolocalización y la validación física de los sitios de acuerdo a las funciones de los comités del CTC y CUACS. (CDM, 2014).

Pressman (2010), establece que las tendencias permiten identificar los criterios de control para administrar la complejidad de la información, acomodar requerimientos emergentes, establecer modelos de proceso que aborden el cambio, coordinar equipos globales con una mezcla de talento cambiante, con el único propósito de poder integrar en un solo entorno el uso de las diversas herramientas informáticas utilizadas para la recopilación de la información durante las fases del Proyecto México Conectado en el Estado de Tabasco. Desde esta perspectiva la integración de la información de los diversos instrumentos utilizados se pueden identificar bancos de información elaborados con la suite ofimática de Microsoft; de forma particular, Word, Excel, Power Point, así como herramientas de gestión para la georreferencia de los sitios e infraestructura propuestos, en la Fig.1 se muestra la estructura general de integración y uso de los datos proporcionados por las diversas dependencias, la cual fue integrada por parte de la IOE para ser expuesto y analizado por parte 
del Comité Técnico de Conectividad (CTC), mismo que tiene la función de recopilar $\mathrm{y}$ concentrar el inventario de sitios e infraestructura de espacios públicos a conectar y utilizar en la entidad. (ICN, 2014). Esta información generada por las dependencias divulga las características esenciales de los sitios, inmuebles, responsables y espacios públicos propuestos para el proyecto, así como la normatividad, transparencia y evidencia de existente por parte de las áreas responsables.

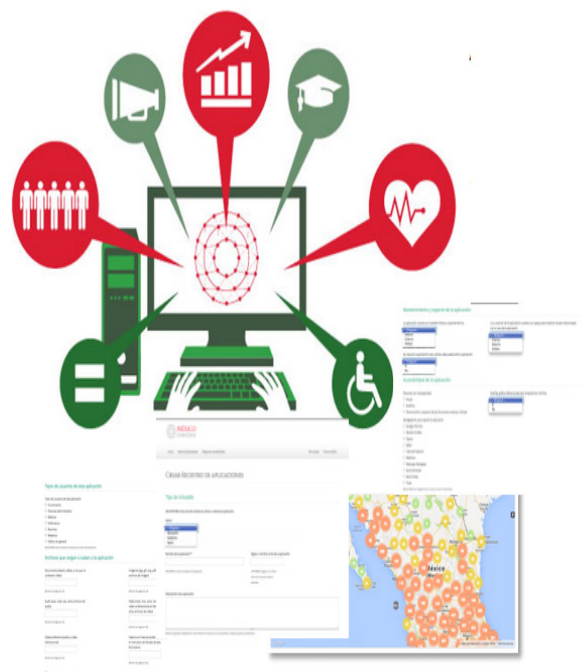

Fig. 1. Esquema general de integración de información. Fuente: Instancia coordinadora nacional, 4 marzo 2014. (ICN, 2014).

Para establecer el procedimiento del análisis y revisión de la información proporcionada por las dependencias y actores participantes es fundamental la comunicación entre los comités, la cual es imprescindible para una correcta toma de decisiones en la consolidación del inventario final de sitios e infraestructura a utilizar y conectar en el Estado, los cuales tienen la facultad de establecer los anchos de bandas requeridos para cada sitio, tipo de torres, aplicaciones a utilizar, filtrados de contenido y distribución por zona, todas estas acciones son propuestos para su aprobación por parte de la mesa de coordinación (MC) junto al inventario final de sitios a conectar en Tabasco. (ICN, 2014).

Dentro de algunos aspectos esenciales en el desarrollo de la fase de planeación, se establece la elaboración del inventarió final de sitios e infraestructura de telecomunicaciones, los cuales contemplan diversos campos que permiten la generación de indicadores, que establecen las actividades a desarrollar por parte de la Instancia Operadora Estatal (IOE), en conjunto con el Comité Técnico de Conectividad (CTC) y el Comité Uso del Aprovechamiento de la Conectividad Social (CUACS). Uno de los primeros resultados para la consolidación del inventario de sitios propuestos en Tabasco, una muestra de 4,754 sitios y espacios públicos los cuales cumple con el proceso de integración y normalización de la información obtenida por parte de todas las dependencias y actores participantes, partiendo de un seguimiento colaborativo que brinda la técnica de trabajo SCRUM y siendo sometidos a dos procesos más de revisión aplicando pequeños Sprint utilizados en el seguimiento del proyecto.

Este primer resultado de información se alcanza aplicando micro actividades especificas a las dependencias y responsables técnicos para identificar los sitios propuestos, aplicando otros procesos de elegibilidad y características especificas de acuerdos a las normas establecidas por parte de la Sociedad del Conocimiento de la Secretaría de Comunicación y transporte para elaborar los instrumentos finales a utilizar para obtener el inventario final de sitios propuestos para el Estado de Tabasco. De igual manera se elaboró un análisis y diseño exploratorio descriptivo, el cual establece la identificación de los criterios finales de elegibilidad de los sitios y espacios públicos del estado de Tabasco partiendo de los resultados obtenidos de la muestra y apoyados bajo la investigación documental de fuentes bibliográficas, estudios técnicos de los sitios y la factibilidad en telecomunicaciones, leyes de los tres órdenes de gobierno y estudios cartográficos relacionados con la investigación. Para la definición de la población de estudio, se estableció como referencia el total de inmuebles adscritos a los tres sectores principales los cuales son: educativo, salud, gobierno, y se consideraron dependencias u órganos desconcentrados de 
la entidad dando como resultado un padrón de 12,147 sitios y espacios públicos a la cual se le aplicaron los dos instrumentos finales para obtener el inventario final del proyecto. La muestra de la investigación propuesta se elaboró listando los sitios y espacios públicos establecidos en el padrón y clasificándolos por el número de población al que benefician. El listado del sector educativo está conformado por 5,675 escuelas y oficinas en todos los niveles, el sector salud con 3,895 centros y el sector gobierno por 2,577 oficinas y espacios públicos, corroborando que los listados de los tres sectores, como se muestra en la Fig.2.

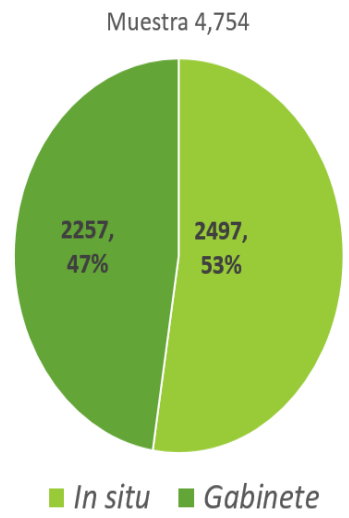

Fig. 2. Muestra establecida para su validación en Tabasco. Fuente: Instancia operadora estatal, 25 de marzo del 2014. (IOE Tabasco, 2014).

La muestra de estudio de 4,754 sitios fue definida de un universo de 12,147, los cuales se deben de validar usando dos instrumentos. El primero es una validación física del inmueble de 2,257 sitios, concentrando información básica para su identificación (fotos, responsable del sitio, georreferencia, domicilio, entre calle, tipo y subtipo de inmueble, dependencia responsable, longitud, latitud, datos de contacto, clasificación del tipo de localidad, tipo de acceso, responsable directo y responsable indirecto, municipio, localidad, tipo de población, numero de habitante, servicios básicos -Luz y seguridad en el inmueble-, región), equivalente al 47\% de la muestra definida por los comités. En la fig. 3. Se muestra parte del instrumento utilizado, herramientas colaborativas y evidencias que describe y validad el sitio o inmueble propuesto para ser propuesto para recibir conectividad de internet.

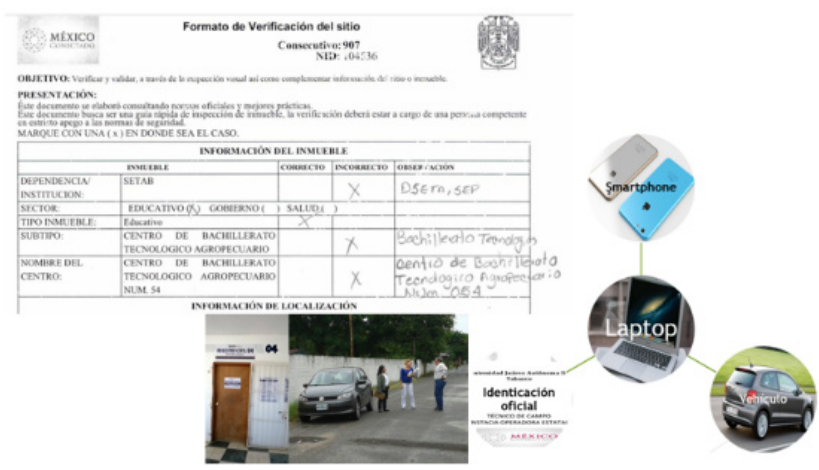

Fig.3. Muestra de la realización de la visita in situ, aplicando los criterios de elegibilidad y validación de los sitios o inmuebles. Fuente: Instancia operadora estatal, 25 de marzo del 2014. (IOE Tabasco, 2014).

Un segundo instrumento, es la validación en gabinete de 2,497 sitios equivalente al 53\%, la cual consiste en utilizar herramientas como google maps, para la identificación de los inmuebles propuestos dentro de la muestra y el resto de los sitios se estableció un mecanismo de integración y depuración aplicando criterios como: Sitios Duplicados, en remodelación, reubicación o encontrarse en inmuebles particulares. Para esta actividad se estableció una tarea de revisión de renglón por renglón la cual busca alcanzar la aplicación de los criterios establecidos por parte de la MC y el CTC. En la Img.1. Se muestra la actividad realizada por personal de la IOE.
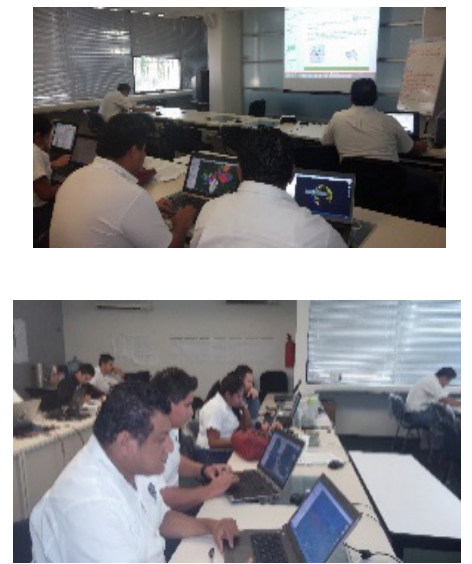

Img.1. Muestra de la realización de la revisión de gabinete, aplicando la tarea de revisión de renglón por renglón. Fuente: Instancia operadora estatal, 25 de marzo del 2014. (IOE Tabasco, 2014).

El padrón se depuro realizando un cruce de la información de las diversas bases de datos integrados por las propuestas de información 
proporcionada por las dependencias para establecer sitios y espacios públicos duplicados, no existentes y que no estuvieran bajos los criterios iniciales de selección para lo cual se diseñó un procedimiento de muestro aleatorio automatizado, asumiendo que la máxima varianza es la población beneficiada y los criterios establecidos por la Mesa de Coordinación (MC) por cada sitio y espacio público, arrojando un universo final de UF=7,272.

Para la realización del estudio de las variables propuestas, se diseñaron como instrumentos dos cuestionarios de selección múltiples. El primer cuestionario con 43 ítems dirigido a los responsables técnicos y operativos de los tres sectores, considerando las propuestas establecidas de elegibilidad por parte de la Mesa de Coordinación (MC) y del Comité Técnico de Conectividad (CTC), los cuales buscan identificar las condiciones físicas en telecomunicaciones existentes en el sitio y la validación de su existencia. El segundo cuestionario de 35 ítems dirigido a los directivos y encargados de los sitios y espacios públicos, con la finalidad de conocer las condiciones físicas del inmueble y su factibilidad técnica y operativa para su inclusión dentro del proyecto. Los cuestionarios fueron probados y ajustados mediante dos ejercicios de recopilación de información, el primer instrumento fue aplicado en una validación piloto de gabinete a un universo de 2,497 sitios y espacios públicos. La validación de los resultados fue a través del CTC, mediante el desarrollo de un análisis estadístico, utilizando Microsoft Excel, en donde se integraron los 43 ítems para su estudio. El segundo instrumento fue aplicado en una visita física a los 2,257 inmuebles y la validación de los resultados fue a través de la MC. Los resultados se validaron con el apoyo de una plataforma electrónica web, desarrollada en Adobe Coldfusion 9, bajo una arquitectura RIA y con una base de datos Oracle $11 \mathrm{~g}$, bajo la herramienta de proceso ágiles Scrum, en donde se establecen las principales reglas de elegibilidad de los sitios y espacios públicos propuestos, con su documentación correspondiente.
Luego de recabada la información de los dos cuestionarios, se procedió a integrar los 78 ítems en una sola base de datos empleando la herramienta Oracle, con el fin de ordenar de forma sistematizada la respuesta de los encuestados e integrar el expediente electrónico de cada sitio y espacio público validado. Los sectores beneficiados dentro del Proyecto México Conectado en la entidad son: Educación, Saludy Gobierno, los cuales se muestran en la Gráfica 1, indicando el número de sitios propuestos por sector, siendo el sector educativo el más beneficiado. De esta último análisis, revisión y validación se conformó el universo final de sitios propuesto para recibir conectividad, la cual da apertura a la fase de licitación estableciendo un volumen de 7,272 sitios y espacios públicos (IOE Tabasco, 2014, págs. p 66 - 68).

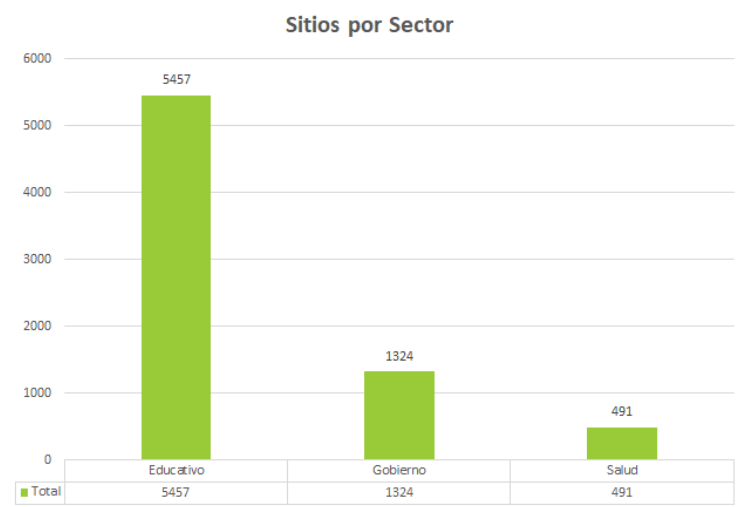

Gráfica. 1. Universo de sitios propuestos por sector en Tabasco. Fuente: Instancia operadora estatal, 9 mayo 2014. (IOE Tabasco, 2014, págs. p 66 - 68).

Esta muestra de 4,754 establecida en el proceso automatizado, se ejerce bajo los requerimientos definidos por parte de los lineamientos del Proyecto México Conectado y de acuerdo con la información proporcionada por parte de los integrantes del Comité Técnico de Conectividad (CTC) y por la aplicación de los instrumentos de validación y despliegue realizados por la Instancia Operadora Estatal (IOE), en donde se registraron y confirmaron hasta en dos iteraciones los 7,272 sitios. En la Gráfica 2. Se muestra los datos básicos utilizados para la validación de los sitios y el despliegue en Recursos utilizados en la primera iteración. (ICN, 2014). 

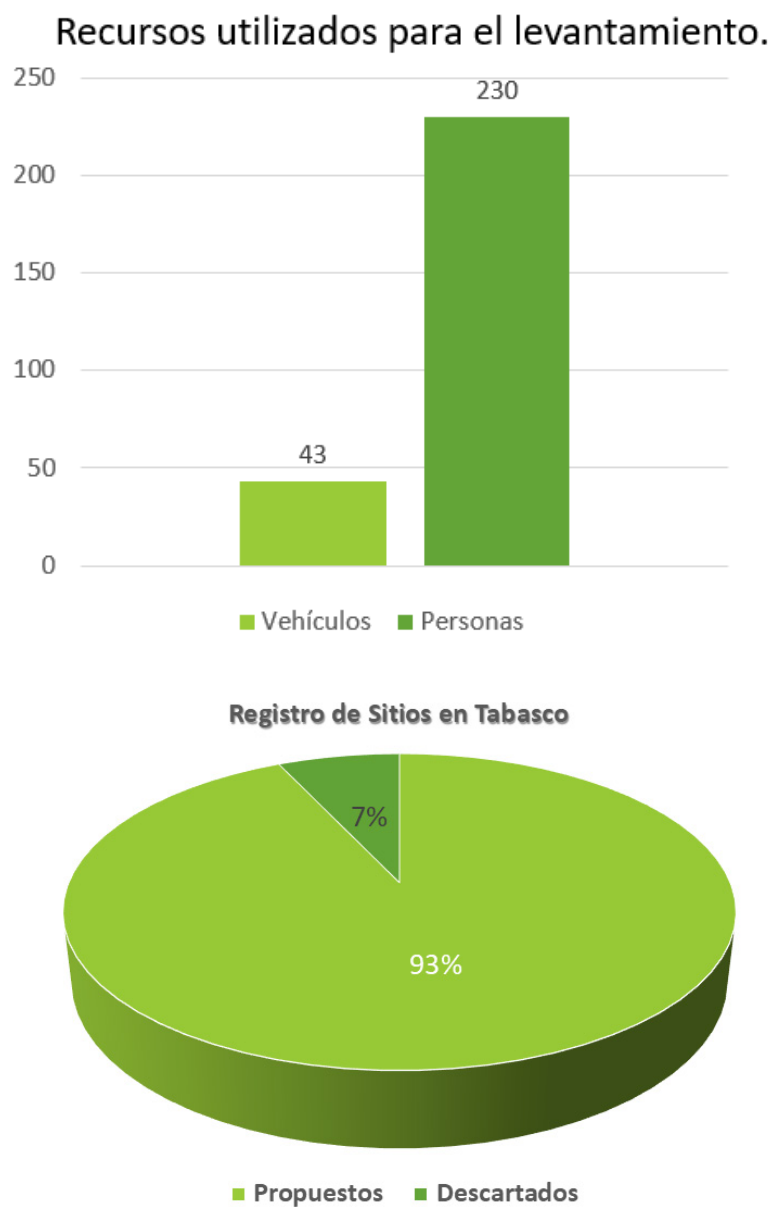

Grafica. 2. Recursos utilizados para la validación de los sitios en la primera iteración del PMC. Fuente: Instancia operadora estatal, 25 de marzo del 2014. (IOE Tabasco, 2014).

De los 7 mil 272 sitios propuesto para recibir conectividad, se identificaron 509 (7\%) cuenta con alguna conexión a internet y 6 mil 763 sitios carecen de este servicio. De conformidad con la cifra presentada, se estima con la implementación del PMC en el Estado de Tabasco, se logrará proveer de conectividad a cada uno de los sitios y espacios públicos que fueron propuesto, lo que permitirá al Estado de Tabasco pasar del lugar 28 nacional a los primero 10 lugares en acceso a internet de banda ancha. En Gráfica 3, se muestra la distribución de sitios y espacios públicos por municipios y por dependencias. (Gobierno de la República G. d, 2014; INEGI, 2014.).
CONCENTRACION DE SITIOS POR MUNICIPIO EN LA LICITACION

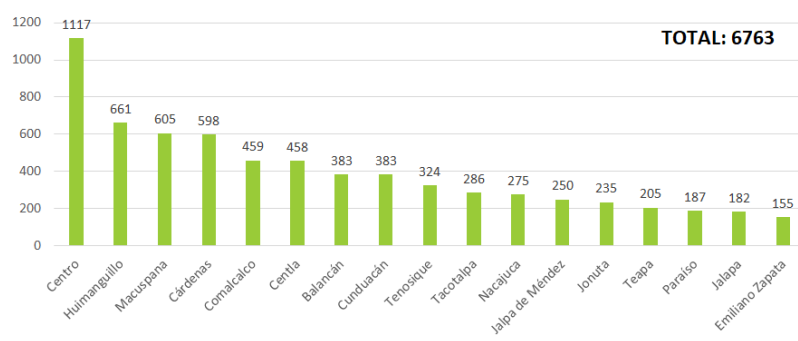

Gráfica 3. Distribución de sitios y espacios públicos por municipios del Estado de Tabasco. Fuente: Instancia operadora estatal, 25 de marzo del 2014. (IOE Tabasco, 2014).

\section{RESULTADOS DE LAINTEGRACIÓNY USO DEL SISTEMA DE INFORMACIÓN TIPO RIA PARA EL CONTROL, SEGUIMIENTO DEL PROYECTO MÉXICO CONECTADO EN EL ESTADO DE TABASCO.}

La información es un recurso vital para toda organización y el buen manejo de esta puede significar la diferencia entre el éxito o el fracaso para todos los proyectos que se emprendan, es por ello que durante la ejecución y el seguimiento del Proyecto México Conectado en el Estado de Tabasco, la información que fluye tiene como objetivo el poder apoyar en la toma de decisiones en todos los niveles de comunicación, para así establecer las mejores estrategias para alcanzar el objetivo planteado del proyecto en la entidad, la cual se centra en reducir la brecha digital en el Estado. En un proyecto de tal importancia para el País y el Estado, es indispensable estructurar la información de tal forma que las actividades realizadas por los colaboradores, áreas, departamentos y dependencias, siempre se encuentre debidamente identificada para poder establecer las acciones a seguir en cada etapa del proyecto. Este principio estructural contempla la implementación de un sistema de información, bajo el estándar de integración y acoplamiento de la información partiendo de un modelo de bodega de datos, la cual permita integrar la fuente de información de todas las dependencias en un solo modelo integral de información. (Pressman R. , 2010), este esquema de comunicación se presenta como una herramienta útil para el 
manejo de toda la información que se genere Esquema 1, reduciendo factores comunes como son: Información dispersa y asilada, Información duplicada o incompleta e Información alterada, la cual no permite realizar una correcta estrategia en la toma de decisión. Estos factores comunes se reducen con la integración de los objetivos que deben de cumplir un sistema de información que son: 1). -automatizar procesos, 2). -proporcionar información que sirva para la toma de decisiones y 3). - lograr una ventaja competitiva a través de su implementación y uso. (Cruz, 2005).

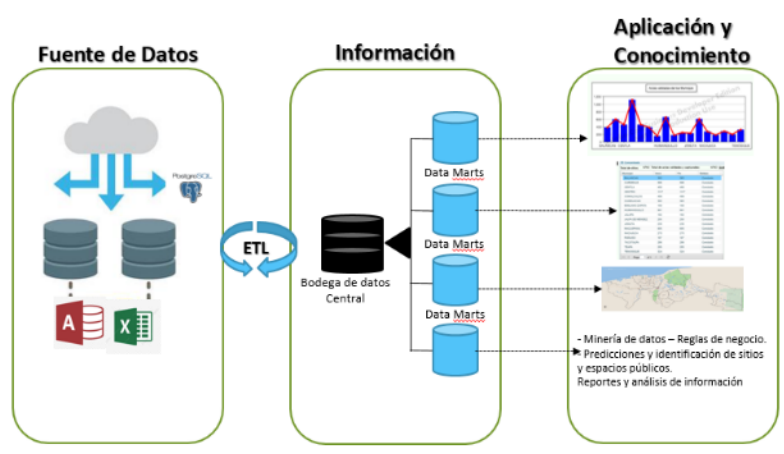

Esquema 1. Esquema de recopilación e integración de la información del proyecto México conectado Fuente: Desarrollo propia.

Según Trigas Gallego, Palacio Juan (2015), la recopilación de la información e identificación de los principales actividades de un proyecto son esenciales para definir su inicio y fin, para lo cual la IOE reestructura sus instrumentos de recopilación e integración de toda la información, clasificándola de acuerdo a las fases de planeación y licitación, se estableció a partir de ello un cronograma de trabajo que permita cubrir las necesidades y reglas de operación en la implementación de los sitios y espacios públicos, integrando las mejores prácticas de la metodología SCRUM, permitiendo identificar las acciones prioritarias para la toma de decisiones en pequeñas tareas involucrado a los diversos actores del proyecto. En la Ilustración 2, se muestra un resumen del plan de acción para el seguimiento de la información de las fases del proyecto.

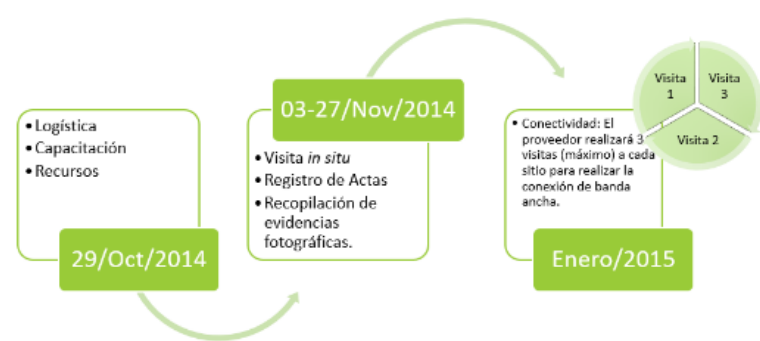

Ilustración 2. Muestra del plan de acción para el seguimiento de la información para la implementación de los sitios y espacios públicos. Fuente: Desarrollo propia.

En definición scrum es un proceso en el que se aplican de manera regular un conjunto de buenas prácticas para trabajar colaborativamente, en equipo y obtener el mejor resultado posible de un proyecto. (Proyectos Ágiles, 2008). El proceso que establece scrum, para el cumplimiento de sus buenas practicas, aplicable a cualquier proyecto. Estas buenas practicas establecieron las reglas de ejecución y desarrollo de la herramienta tecnológica necesaria para la Instancia Operadora Estatal (IOE), la cual concentrará la información de las actividades diarias desarrolladas por cada uno de las áreas, dependencias y comités que tenga alguna participación en el Proyecto México Conectado.

Este esquema de trabajo se estableció definiendo las actividades principales en donde la IOE concentraba la mayor información recopilada por parte de los dos comités rectores del Proyecto México Conectado y por parte de las tareas establecidas en la Mesa de Coordinación. De igual forma, se integraron las actividades establecidas por parte de la ICN, la cual establece las acciones a realizar durante el desarrollo de las fases implementación y ejecución del proyecto. (IOE Tabasco, 2014). En la Ilustración 3, se establece en concordancia a la aplicación de la metodología Scrum un nuevo orden de integración de la información acoplando las variables prioritarias de los comités y definiendo nuevos canales de comunicación y seguimiento en la operatividad final del proyecto. 


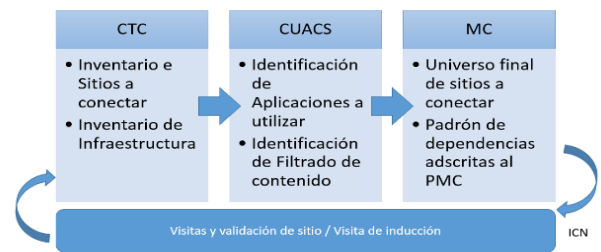

Ilustración 3. Nuevo Esquema de recopilación de la información del proyecto México conectado

Fuente: IOE Tabasco, 2014.

El resultado de este esquema de trabajo, permitió la identificación de los principales universos de almacenamiento que existía dentro de la IOE de Tabasco, priorizando con ello las acciones de desarrollo. El desarrollo del modelado de datos se fundamentó en la integración de todos los archivos de Excel, base de datos externas y servidores remotos existentes en cada actividad solicitada a la IOE, lo cual permitió construir un mapa conceptual robusto para las necesidades específicas de toma de decisión dentro de la IOE. En el Diagrama 1, se muestra el esquema de base de datos empleado para el desarrollo de la aplicación web usando herramientas RIA, que permiten tener la información actualizada instanciada desde cada dependencia participante y así establecer una toma de decisiones diferente, dentro de un proceso sistematizado durante el desarrollo del proyecto en la entidad.

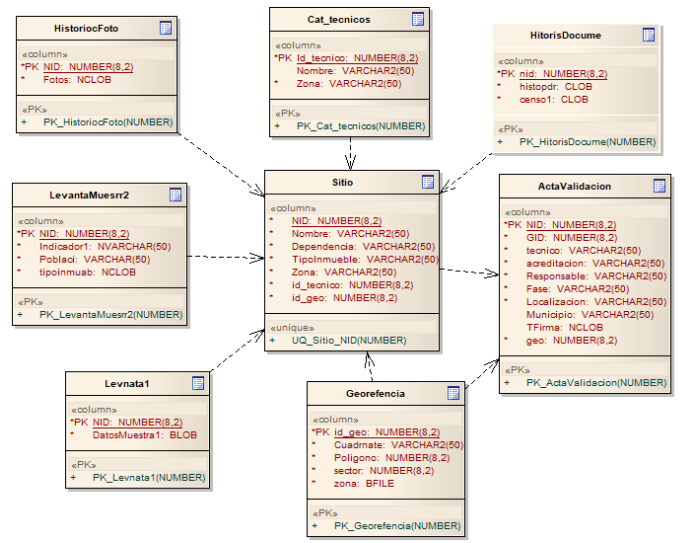

Diagrama 1. Esquema conceptual, referente al modelado de los datos y su integración con los diversos universos de información. Fuente: Desarrollo Propio.

El desarrollo de las interfaces del sistema de información el cual concentra soluciones técnicas y métodos de aplicaciones tipo RIA, usando framework de usabilidad y acoplamiento de integridad de información, las cuales permiten realizar el agrupamiento de las acciones divididas por cada una de los municipios de la entidad, definiendo a los sitios y espacios públicos de acuerdo a su naturaleza y su totalizado. En la Interfaz 1, se muestra el entorno sistematizado del agrupamiento de sitios. Partiendo de este agrupamiento se presenta un mapa de la cobertura por sitios y espacios públicos, estableciendo un criterio de acción mediante la identificación de colores para puntualizar el estatus del mismo, donde el Café=Sitio no localizado o existen algunos elementos que no permiten definirlo con claridad para establecer su servicio, el Verde $=$ Sitio localizado, censado y disponible para recibir el servicio de internet, así como una gráfica de incidentes relacionado con el mismo de igual forma se visualizan uno de los 17 mapas utilizados para la semaforización de la información y mediante este entorno gráfico clasificar el estatus del sitio.
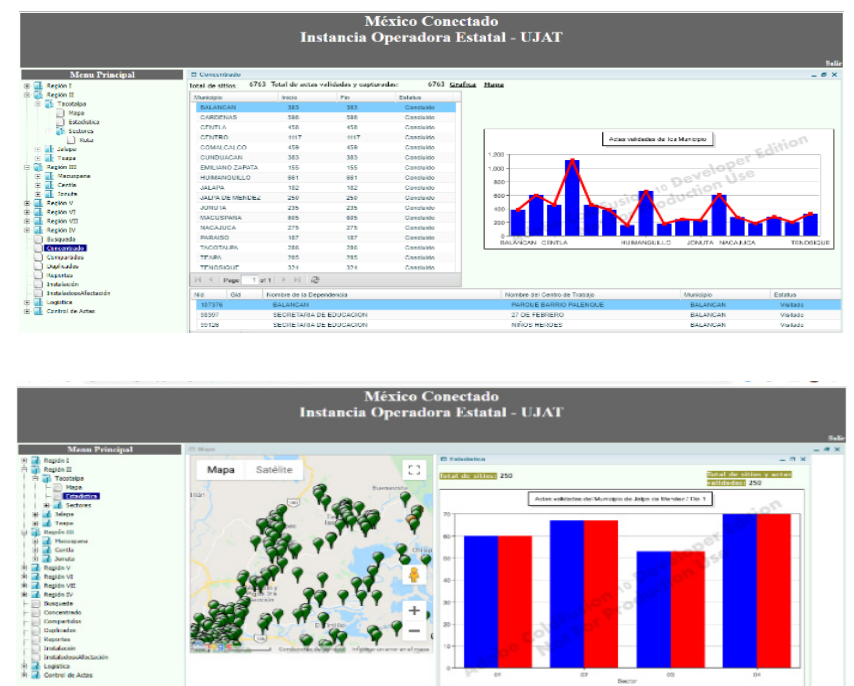

Interfaz 1. Interfaz de agrupación de los Sitios y Espacios públicos por entidad, sector y totalizado.

Fuente: Desarrollo Propio, obtenida del sistema de información de la Instancia Operadora Estatal.

En otro de los apartados del Sistema de Información se integra la información técnica del Sitio o Espacio Público, el cual contempla su identificador, su acta electrónica, las evidencias fotográficas del inmueble, así como el tipo de incidencias de acuerdo a los criterios de selección por parte de la $\mathrm{MC}$, permitiendo con ello acceder 
a datos tales como: El responsable del sitio, su identificación, teléfono, formas y métodos de localización y población beneficiada del servicio de internet a un rango aproximado de cobertura de 25 a 30 metros a la redonda del punto central del sitio. En la Interfaz 2, se exponen los módulos correspondientes a la integración de la información técnica del sitio.
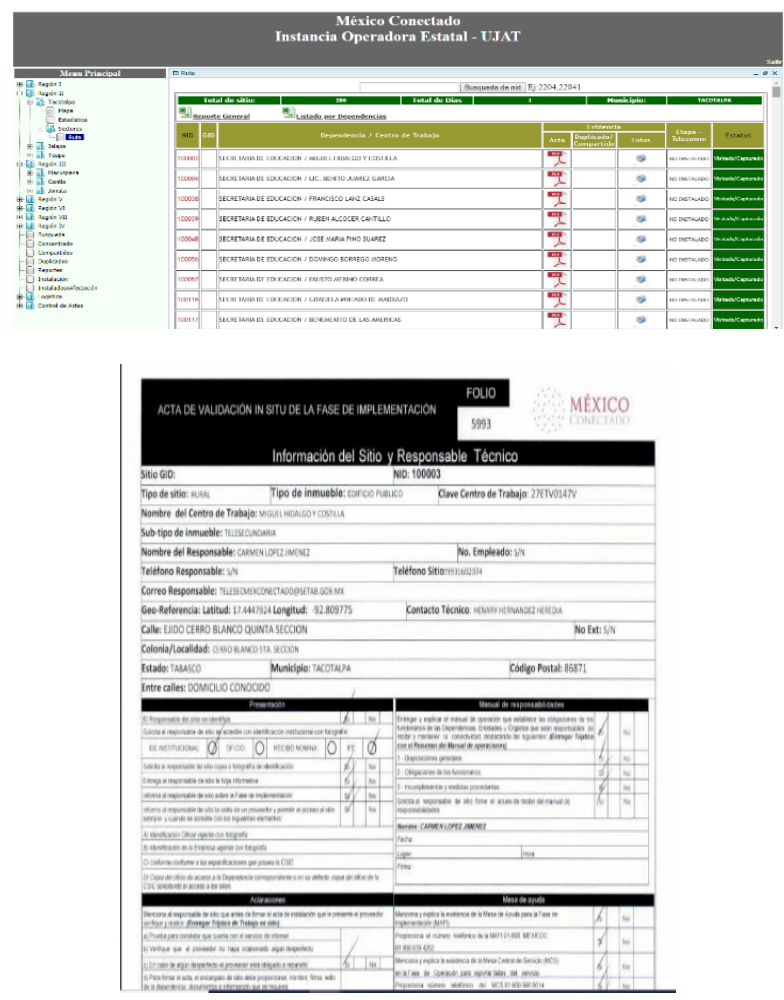

Interfaz 2. Información técnica del Sitio. Fuente:

Desarrollo Propio, obtenida del sistema de información de la Instancia Operadora Estatal.

En el apartado correspondiente a la asociación del banco fotográfico de evidencias relacionado a la identificación, liberación e instalación del sitio y espacio público se encuentran datos como: Fotos del inmueble identificado, cartel o etiqueta que indique que existe cobertura de internet gratuito de forma inalámbrica y para la dependencia mediante el uso de switch de conexión, a partir de un tiempo de terminado, establecido por la SCT, de 30 min por conexión por usuario. En la Interfaz 3 , se muestra los módulos correspondientes al uso de los bancos fotográficos de evidencias y el soporte de información referente al sitio o espacio público correspondiente.
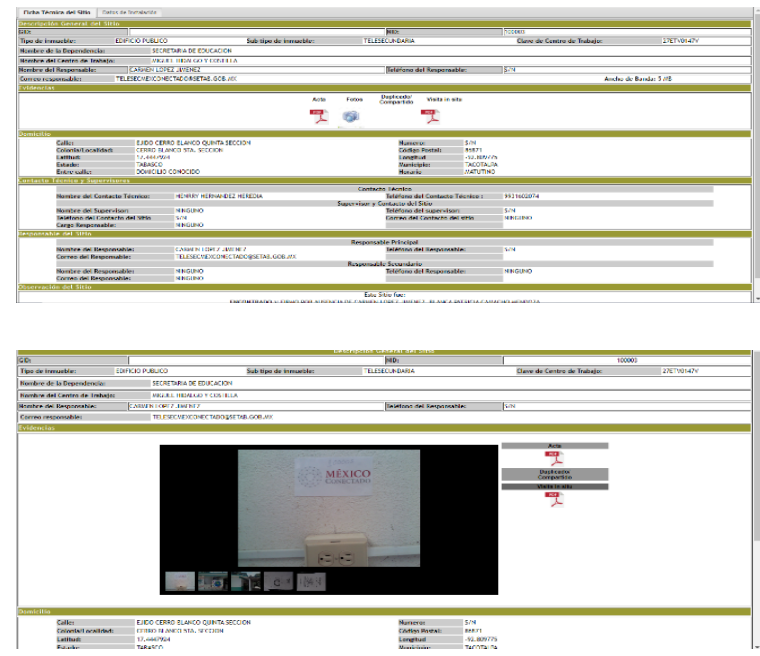

Interfaz. 3. Módulos de uso y seguimiento del Banco fotográfico de evidencia del Sitio. Fuente: Desarrollo Propio, obtenida del sistema de información de la

Instancia Operadora Estatal.

El reporte de incidencia dentro del Sistema de Información se clasifica a partir de 2 rubros, uno el número del incidente por sitio o espacio público, la asignación del integrador responsable para su atención, el cual debe de estar cubierto en primera instancia dentro de las 72 horas posteriores al reporte emitido y liberado por conducto de las mesas de ayuda establecidas por parte de la SCT, para garantizar la persistencia del servicio. En la Interfaz 4, se presentan los módulos de control para el seguimiento de incidencias y sus evidencias de solución.
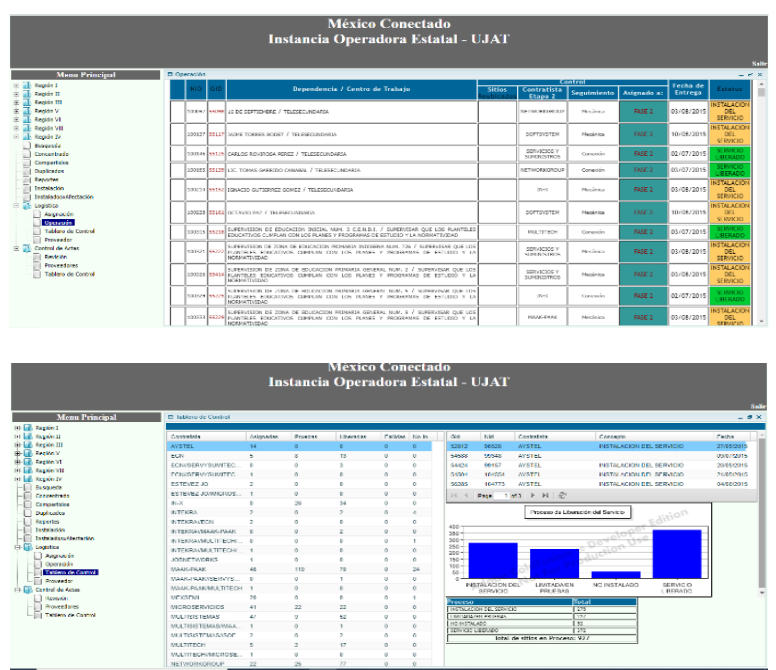

Interfaz 4. Información técnica de reporte de incidencias reportadas. Fuente: Desarrollo Propio, obtenida del sistema de información de la Instancia Operadora Estatal. 


\section{CONCLUSIONES}

Al terminar el análisis, diseño y sistematización de las variables de los diversos bancos de información integradas en cada una de las fases del proyecto y la utilización de los datos obtenidos en la ejecución de los dos instrumentos aplicados a los responsables de los sitios y espacios públicos registrados, se observó que a partir del uso del Sistema de Información se detectaron con mayor rapidez dos de los factores con mayor recurrencia en los incidentes al momento de aplicar las reglas de exclusión, los cuales fueron omitidos en las fases iniciales, tales como son la falta de energía eléctrica y de seguridad en los inmuebles, lo que permite establecer un precedente de alto riesgo en la instalación del servicio, incluyendo una variable más para ser utilizada para fortalecer en los próximos años por parte de las autoridades. El margen de exclusión de los inmuebles es del $38.8 \%$, distribuido de la siguiente forma: sector educativo 1,124 inmuebles, sector salud 559 y sector gobierno de 945 .

Con el uso del Sistema de información se incentiva un mejor control en el seguimiento de los sitios y espacios públicos, en específico en una de las actividades más recurrente, como lo es, el cambio de responsables, reubicación del inmueble y en la identificación de zonas vulnerables a factores externos no establecidos en los criterios de selección, tales como son: inundaciones, bloqueos, afectaciones por la industria petrolera establecidas y por los altos índices de inseguridad en la entidad.

Se estima, de acuerdo a los datos establecidos en el Sistema de Información y partiendo del universo final propuesto de 6,763 que el 61.1\% de los inmuebles y sitios, validados, aceptados y licitados, equivalentes a 4,135, los cuales sean provistos del internet de México Conectado y entren en operación en los próximos meses, traduciéndose esta inclusión tecnológica en beneficios sociales y económicos para el Estado, colocando a la entidad dentro de los diez primeros lugares de accesibilidad al ciberespacio en el país. En lo referente al ámbito social, la población directamente beneficiada, se verán transformaciones digitales tales como la aplicación de gobierno electrónico, telemedicina, Educación en línea y servicios indirectos hacia las comunidades como son intercambio cultural, aprendizaje colaborativos multi-idiomas, acceso a las redes de conocimiento, redes sociales, canales de videos y un nuevo enfoque centrado en la culturización tecnológica en zonas profundas, donde aún presenta este tipo de rezago; y en lo económico en la reducción de los costos de los servicios al incentivar competencia en el sector de las telecomunicaciones.

Con la información integrada y normalizada se proyecta el crecimiento tecnológico por cada región en el Estado de Tabasco, brindando con ello un mapa de los recursos tecnológicos asignados y existentes en la región, estableciendo a partir de este instrumento un marco de referencia y guía para evitar duplicar recursos y fortalecer zonas donde aún no se cuenta con algún tipo de servicio tecnológico a beneficio de la sociedad. Esto permite medir la obsolescencia de los recursos tecnológicos implementados y su posible mantenimiento o reemplazo para seguir brindando un servicio de mejor calidad a beneficio de la sociedad.

A partir de la información integrada en el banco de datos central del proyecto, se diseñan una variedad de indicadores en apoyo al seguimiento, control y administración del uso del servicio de internet por cada sector, región y municipio, proyectando escenarios de crecimiento en el ancho de banda requerido, el tipo de población: Rural, Urbana y Suburbana, discapacidades, analfabetismo y pobreza, entre otros, los cuales son un referente para establecer estrategias de inclusión e integración social, altamente necesarias en la actualidad. 


\section{REFERENCIAS}

1. Adobe System. (2016). Soluciones de creatividad, marketing y gestión de documentos. (Adobe, Ed.) Recuperado el 9 de 02 de 2016, de https://www.adobe.com/la/aboutadobe/pressroom/pr/ feb2008/RIA_Launch_SP.pdf: www.adobe.com

2. Burgos Aguilar, J. V. (2013). Motivaciones de uso de los recursos educativos abiertos en la práctica docente. Competencias Docentes y Prácticas Educativas Abiertas en, 214 - 254.

3. CDM. (2014). Lineamientos del Proyecto México Conectado. (CSIC, Productor, \& CSIC) Recuperado el 09 de 02 de 2016, de http://mexiconectado.gob.mx/images/archivos/2013_09_27 Lineamientos_Mexico_conectado.pdf: http://mexicoconectado.gob.mx/

4. CEMABE. (2014). Censo de Escuelas, Maestros y Alumnos de Educación, Básica y Especial;. México: CEMABE.

5. CEPAL. (2015). "Estado de la banda ancha en América Latina y el Caribe”. Obtenido de http:// repositorio.cepal.org/bitstream/handle/11362/38605/S1500568_es.pdf;jsessionid=532D73BAB8 776C9FEE3CCEB56556A2E2? sequence $=1$

6. CEPAL. (2016). Estado de la banda ancha en América Latina y el Caribe. Obtenido de Recuperado de https://www.cepal.org/es/publicaciones/40528-estado-la-banda-ancha-americalatina-caribe-2016

7. Compranet. (2014). Servicios de Internet en sitios públicos en los estados de Colima y Tabasco. Recuperado el 01 de 03 de 2016, de Acta electrónica, correspondiente al acto de fallo de la Licitación LA-009000937-N11-2014: https://compranet.funcionpublica.gob.mx/web/login.html

8. Cruz, S. (2005). Importancia de los sistemas de información para las Pymes, en linea GestioPolis - Conocimiento en Negocios. Recuperado el 03 de 03 de 2016, de http://www.gestiopolis.com/ importancia-sistemas-informacion-pymes/: http://www.gestiopolis.com/

9. CSIC. (2012). Covocatoria a la Licitación Pública Nacional Electónica LA-009000937-N14-2012. Ciudad de Mèxico: SCT.

10. CSIC. (2013a). Lineamientos del Proyecto México Conectado. Ciudad de México: SCT.

11. CSIC. (2018a). Servicios Contratados - Libros blancos Proyecto México Conectado. México: SCT.

12. CSIC b. (2014). Convocatoria a la Licitación Pública Nacional Electrónica LA0090000937-N11-2014. Ciudad de México: SCT: SCT.

13. CSICa. (2018). Servicios contratados - Libro Blanco del Proyecto México Conectado. Ciudad de México: SCT.

14. Cuesta, F. (12 de 10 de 2008). La realidad de la empresa virtual. Obtenido de http://observatorio. digital/blog/realidad-virtual-empresas/

15. Diputados, C. d. (2013). Inicitiva del Titular del Poder Ejecutivo, con proyecto de decreto, que refroam y adiciona diversas disposiciones de la Constitución Política de los Estados Unidos Méxicanos, en materia de telecomunicaciones. México: Gaceta Parlamentaria: http:/gaceta. diputados.gob.mx/PDF/62/2013/mar/20130312-II.pdf.

16. DOF. (2013). Constitución Política de los Estados Unidos Méxicanos - Decreto de reforma en materia de telecomunicaciones. Obtenido de obtenido de DOF: http://www.dof.gob.mx/nota detalle.php? codigo $=5301941 \&$ fecha $=11 / 06 / 2013$

17. DOF. (2014). Ley Federal de Telecomunicaciones y Radiodifusión. Obtenido de Obtenido de http://www.dof.gob.mx/nota_detalle.php?codigo $=5352323 \&$ fecha $=14 / 07 / 2014$

18. García - Alsina, M. (2017). Big Data. Gestión y Explitación de Grandes Volúmenes de Datos (Libro en Papel). UOC, S.L.

19. Gobierno de la República, G. (2013a). Estrategia Digital Nacional. Ciudad de México: Gobierno Federal.

20. Gobierno de la República, G. (2013b). Primer Informe de Gobierno 2012-2013. México: Republica Méxicana.

21. Gobierno de la República, G. (2014b). Programa Nacional de Infraestructura 2014 - 2018. México: SHCP.

22. Gobierno de la República, G. (2015a). Tercer Informe de Gobierno 2014 - 2015. Obtenido de Obtenido de http://www.presidencia.gob.mx/tercerinforme/

23. Gobierno de la República, G. d. (2014c). Primer Informe de Ejecución del Plan Nacional de Desarrollo 2013 - 2018. Obtenido de Obtenido de: http:/www.sct.gob.mx/fileadmin/ 
DireccionesGenerales/DGP/PDF/SCT-1erIE-2013.pdf

24. Gómez Navarro, D. (2018). La Brecha Digital: Una revisión Conceptual y aportaciones metodológicas para su estudio en México. EntreCiencias - Dialogos entre la sociedad del Conocimiento, Entreciencias 6(16):49-64 Abr - Jul 2018.

25. Histinf.blogs.upv.es. (2016). Historia de la web 2.0|Historia de la Informática. Recuperado el 10 de 02 de 2016, de http://histinf.blogs.upv.es/2010/12/12/historia-de-la-web-2-0/: http://histinf. blogs.upv.es/

26. ICN. (2014). Informe Ejecutivo de la Mesa de Coordinación del Proyecto México Conectado en Tabasco. Universidad de Guadalajara, Instancia Coordinadora Nacional. Villahermosa, Tabasco: PMC.

27. INAFED. (2010). Enciclopedia de los Municipios y Delegaciones de México Estado de Tabasco. Obtenido de http://www.inafed.gob.mx/work/enciclopedia/EMM27tabasco/regionalizacion.html: /www.inafed.gob.mx

28. INEGI. (2014). "Encuesta nacional sobre disponibilidad y uso de tecnologías de la información en los hogares 2014". Ciudad de México: INEGI.

29. IOE Tabasco. (2014). Fase de Planeación - Estudio de Sitios y Espacios públicos propuestos a recibir conectividad. Villahermosa, Tabasco: PMC.

30. IOE Tabasco. (2014). Informe Ejecutivo del Proyecto México Conectado en Tabasco. Universidad Juárez Autónoma de Tabasco, Dirección del Centro de Cómputo. Villahermosa, Tabasco: PMC.

31. IOE Tabasco. (2015). Informe del Avance de la Implementación de los sitios en el Estado de Tabasco. Universidad Juárez Autónoma de Tabasco, Instancia Operadora Estatal. Villahermosa, Tabasco: PMC.

32. Kavanagh, P. (2004). Open Source Software: Implementation and Management (Software Development) 1st Edition. Digital Press; 1 edition (August 9, 2004).

33. Mdirector. (01 de 08 de 2018). https://www.mdirector.com/sms-marketing/tipos-sms.html. Obtenido de https://www.mdirector.com/sms-marketing/tipos-sms.html: https://www.mdirector. com/sms-marketing/tipos-sms.html

34. Monte Galiano, J. (2016). Implementar SRUM con Éxito. UOC (Oberta UOC Publisih, SL).

35. Pactoporméxico. (2014). Reforma en materia de Telecomunicaciones. Recuperado el 16 de 03 de 2016, de http://pactopormexico.org/reforma-telecomunicaciones/

36. Palacio, J. (2007). Flexibilidad con Scrum. Safe Creative.

37. Palacio, J. (2015). Gestión de Proyectos con Scrum Manager. RIGHTS INFO.

38. Pérez Martínez, E. . (2015). Desarrollo Aplic.Mediante El Framework D. RA-MA S.A. Editorial y Publicaciones (1 de septiembre de 2015).

39. Piña Gutierrez, J. (2018). Informe de actividades 2018. Villahermosa, Tabasco: Justo Sierra.

40. Pressman, R. (2010). Ingeniería de Software Un enfoque práctico - septima Edición pag 712-722. Mc -Graw hill.

41. Pressman, R. (2010). Ingenieria de Software Un enfoque práctico - Septima Edición. Pag. 4. McGraw Hil.

42. Proyectos Ágiles. (2008). Proyectos Ágiles - Scrum. Recuperado el 03 de 05 de 2014, de https:// proyectosagiles.org/que-es-scrum/: https://proyectosagiles.org/

43. R., Lozano, C., \& Ramirez M., S. (2010). Apropiación tecnológica en profesores que incorporan recursos educativos abiertos en educación media superior. Revista Mexicana de investigación educativa, 15(45), 487-513.

44. Ramírez, M. S. (2010). Recursos educativos abiertos en ambientes enriquecidos con tecnología: Innovación en la práctica educativa. México: México: Lulú editorial digital.

45. Rivera Sanchez, C. (2018). ¿Qué es México Conectado? Obtenido de Obtenido en https://www. infotecarios.com/que-es-mexico-conecta/\#.XcG9ZShKjIU

46. SCT. (2014). Red Compartida - El proyecto de Telecomunicaciones más grande en la historia de México. Ciudad de México: SCT.

47. Torres, Díaz, J. (2012). Integración de redes sociales y entornos virtuales de aprendizaje. Rescatado del portal web http://www.um.es/ead/red/35/torres_et_al.pdf. RED. Revista de Educación a Distancia. Número 35.

48. Trigas Gallego, M., \& Domingo Troncho, A. (2015). Gestión de Proyectos Informáticos Metodología Srum. Obtenido de Consultado el dia 10/01/2019, http://openaccess.uoc.edu/ webapps/o2/bitstream/10609/17885/1/mtrigasTFC0612memoria.pdf 